\title{
Compromising algorithmicity and plasticity in autonomous agent control architectures : The autonomous cell
}

\author{
Elpida S. Tzafestas $\underline{(\text { NOTE *) }}$ \\ Intelligent Robotics and Automation Laboratory \\ Electrical and Computer Engineering Department \\ National Technical University of Athens \\ Zographou 15773, Greece. \\ brensham@softlab.ece.ntua.gr \\ http://www.softlab.ece.ntua.gr/ brensham
}


Abstract. While algorithmic autonomous agent control architectures demonstrate high efficiency, they suffer from network structure rigidity that shows in the liability to crucial errors. On the other hand, the redundancy inherent in most connectionist architectures allows for continuous self-organization that compensates for limited-scale neuron failures. In this work, we are seeking to compromise algorithmicity and plasticity in front of local network failures, by extending a basic algorithmic cell model. The extension is twofold : on the one hand we introduce motivation to the cell level, which shows as preference to consume some kinds of messages, while on the other hand we introduce sociality, which shows as adaptivity of the cell to the motivations of its neighbors. Unlike usual connectionist models, there are no connections between cells, but message buffers shared by all cells of a level ; this way, cells can be viewed as floating in a common interaction medium and competing with one another. Another important feature of this arrangement is the necessity of an immune system, i.e., a population of cells that recognize and eliminate the messages that might be detrimental to the integrity of the cellular agent. The model's self-organizational potential is illustrated on the example case of a navigation system. Our simulation results show that the cellular network exhibit plasticity and recover from various types of failures by "discovering" alternative message flow pathways and that multiple failures slow down the system's responsiveness to external events. Issues such as selectivity and the role of diversity are also discussed.

Keywords Cellularity, algorithmicity, plasticity, fault-tolerance, adaptation, self-organization.

Autonomous agents research within the field of behavior-based artificial intelligence and artificial life is concerned, among other things with the exploration and development of mechanisms that allow autonomous agents to exhibit fundamental cognitive abilities. From an architectural viewpoint, such autonomous agents have distributed control systems, either "algorithmic" (as exemplified by Brooks 1986) or "connectionist" (as exemplified by Verschure et al. 1992).

The algorithmic approach (Brooks 1986,1990; Arkin 1987,1989; Steels 1994a) presumes that the problem (usually, a robotic task) is given in advance and that the control system may be built on this 
basis. This approach allows the programming and implementation of extremely efficient, low-cost distributed control structures (Brooks 1991a,b), while the designer maintains a sufficient understanding of the system's algorithmic operation. The price to pay is the rigidity/inflexibility of the network structures involved, in front of apparently similar but not identical tasks. Besides, while the performance of the system degrades rather gracefully when individual components degrade, there is no possibility to recover from such a failure. (NOTE 1) On the other hand, the connectionist approach (Verschure et al. 1992, Husbands et al. 1993, Yamauchi \& Beer 1994) presumes limited "a priori knowledge" about the problem, so that the control system has to learn by itself the specific properties of the problem and somehow converge to its solution. This paradigm relies on redundant network structures with uniform component functionalities, so that recovery from small scale failures is possible. However, and besides inefficiency, redundancy and uniformity lead to lack of understanding of the internal functioning of the system and as a consequence make scalability largely impossible.

From a functional point of view, plasticity is the self-organizational possibility of the network, whether the structure of the network remains fixed (in which case it is called topology-preserving selforganization) or not. The latter case refers to approaches that use a growth mechanism or equivalent (Elman 1993, Cangelosi et al. 1994, Gruau 1995).

Plasticity - Functional viewpoint: Plasticity is the property of a network to selforganize (learn) in response to unforeseen situations, or else its property to adapt to unforeseen input conditions, by giving novel viable responses.

Our long term goal is twofold :

- to reveal organizational principles of distributed control systems that combine the advantages of both approaches, i.e., the efficiency and understandability of the algorithmic approach with the plasticity of the connectionist approach,

and

- to understand the nature of plasticity in the opposite sense as the one given so far, that is a form of plasticity necessary for reasons intrinsic to the network and that do not necessarily coincide with an observer's point of view. This sort of causal plasticity ought to be finally capable of enlarging the application "domain" of the algorithm implemented by the network, i.e., of the class of problems that it can solve, and therefore be the true cause of functional plasticity. 
Distributed control architectures may be structurally analyzed and classified according to two dimensions, the homogeneity or heterogeneity of transfer functions across network components, and the type of network connectivity that may be fixed or plastic. Traditional connectionism (from which architectures such as this of Verschure et al. (1992) are derived) is based on functional homogeneity coupled with connection plasticity. On the other hand, the majority of algorithmic approaches (for instance, Brooks 1986; Chapman 1990; Steels 1994b) is based on connection rigidity, regardless of whether the transfer functions are homogeneous or not. Note that there are neural architectures with fixed connectivity, hence of algorithmic nature (for instance, Beer et al. 1989; Beer \& Chiel 1991; Cliff et al. 1993). In these architectures, each of the neurons accomplishes a very precise function and connections are specific and certainly not "uniform".

Connectionist or neural approaches presuppose homogeneous transfer functions and homogeneous connectivity (in terms of semantics of messages) for all network components; this property allows for observable functional plasticity. On the other hand, non-neural approaches are based on the interconnection of specialized components with the aid of connections having various semantics, what makes such control systems efficient and understandable. For instance, in the subsumption architecture (Brooks 1986), there are three types of connections : the "classic" connection (sending a message), the inhibitory connection and the suppression connection. On the contrary, in the neural paradigm, the variation of connections weights allows a "semantic" variation, the connections remaining structurally homogeneous.

\begin{tabular}{|c|c|c|}
\hline & Fixed & Plastic \\
\hline Homogeneous & Cliff, Beer & Verschure \\
\hline Heterogeneous & Brooks & Cellularity (cf. below) \\
\hline
\end{tabular}

Table 1 Structural classification of distributed control architectures according to two dimensions : homogeneity or heterogeneity of transfer functions across network components, and type of network connectivity that may be fixed or plastic.

To achieve simultaneously algorithmicity and plasticity, it seems therefore logical to combine the transfer function heterogeneity of non-neural approaches with the structural homogeneity of connections of neural ones :

Cellularity is defined as the mode of network organization in which the transfer functions are heterogeneous across components, called cells, but the connections syntax is homogeneous, i.e., the form or structure of all the messages traveling in the connections is the same and the cell cannot treat different connections differently. 
Any semantic variations will show in the way messages are interpreted by different

cells. Additional comments on the messages form may be found in section 3.1.

Usual connectionist architectures fall into this category as well, since they correspond to the degenerate case of homogeneous transfer functions.

We answer to our second goal, by defining plasticity as the property of a network to recover from local cell failures and to maintain itself as a coherent algorithmic activity unit.

Plasticity - Causal viewpoint : Plasticity is the property of a network to self-organize in order to maintain itself as a coherent unit of innate algorithmic activity, that is the property of a network to maintain the relations between its parts.

Plasticity is hence viewed as a sort of redundancy and is analyzed here from a "negative" point of view : what kind of redundancy do we need so that the control system succeeds in solving the given problem despite hardware failures and what cascade of failures should there occur to destroy the control system's operationality? Let us not forget that in healthy living organisms, such as a human or animal body, there are cells dying everyday : in spite of this natural degradation mechanism, organisms manage to survive and even to improve their operation. For an external high-level observer, such as an ethologist, the overall functioning of the organism is not affected by low-level failures. The implementation of a distributed control system on distributed hardware should demonstrate the same property. In a forthcoming study we also plan to analyze this plasticity from a "positive" viewpoint : if there are no failures, what role might redundancy serve and what would it take for the control system to "discover" new problems to solve, i.e., to enlarge the class of problems that it can solve ? Plasticity appears therefore as the cognitive potential that is little by little "exploited" by the agent itself (I am attracted by the idea that learning might just be the reaction of otherwise idle cells that want to participate to the control process).

Another motivation behind this line of work has been the intuitive will to adopt a biology-inspired systemic view (Varela 1979) of autonomous agents, i.e., one that presupposes the same organizational principles at all levels : the cell, the organism, the society and so forth. In (Tzafestas 1995d) we explored computational models of motivation, perturbation, adaptation and sociality/cooperation. This article investigates those issues at the autonomous cell level, i.e., one level below that of the autonomous (cellular) agent. 
The task that we are going to study under the prism of plasticity is the navigation of an agent in an environment containing obstacles to avoid and a line to follow eventually in order to arrive to a given goal-position. Our goal in the remaining of the paper will be to develop a mechanism based on cellularity and plasticity as defined and also to explore its consequences in terms of network structure. The simulations performed and the results obtained have a qualitative value : they show why and how such a mechanism of hardware plasticity is useful and elucidate its properties. In a forthcoming implementation, we plan to actually use this mechanism and the presented cellular model to account for spatial learning of our simulated agent. For the time being, the agent does not learn anything new on its environment, but only how to use best its cellular potential to "implement" its otherwise fixed navigation algorithm.

A final question, up to now neglected, concerns the nature and the origin of failures :

Question : If plasticity is in fact the property of a network to self-organize in front of unforeseen failures/lesions, where do these lesions come from?

We postulate that those failures may in fact be the result of natural degradation process of the agent (even if this degradation is not in the same sense as the wear of man-made machines), i.e., the result of an aging or senescence process : this hypothesis is analyzed in (Tzafestas 1996) where a senescence mechanism is proposed and described.

We will present first the autonomous cell model and we will discuss its properties before proceeding to a demonstration on the navigation problem. Next, we will show why an immune system is necessary as a defense mechanism against persistent perturbations and we will apply those ideas on the same navigation problem.

We are summarizing below the relevant aspects of the basic algorithmic cellular model (see the third chapter of (Tzafestas 1995d) for a more complete description).

- The cell is the elementary unit of storage and information processing. A cell represents and corresponds to a piece of hardware capable of some minimal storage and processing. It has a number of input connection lines, a number of output connection lines, a static or dynamic memory 
vector and a processing unit, that corresponds to a transfer function plus some side-effect updating of the memory vector (adaptation). Cells are therefore structural primitives differentiated by design or by adaptation in a specific context and represent a uniform substrate for building complex aggregates. The cell's complexity lies between that of a simulated neuron and an augmented finite state machine (AFSM, Brooks 1986), since the transfer function is heterogeneous across different cells, but involves exclusively numeric variables and no symbolic states. The transfer function and the size of the memory vector may vary from cell to cell.

- Cells are categorized into functionally different types. There are three types of cells, sensor or input cells, actuator or output cells and processing cells. Sensor and actuator cells are responsible for primitive sensing or actuation operations, respectively. All other cells are internal processing cells.

The cellular architecture is organized hierarchically and in a generally feedforward manner, by successive layers of cells, corresponding to different algorithmic stages. Let us assume the intermediate cellular level shown in figure 1. Assume that cell A breaks. For the algorithm to keep functioning, another cell, either B or C, should undertake A's role. For this to be possible, B or C has to fulfill the following three requirements :

- It should be able to switch to A's transfer function,

- It should be able to recognize and process the messages previously processed by A, and

- It should be able to continue doing its previous work despite undertaking also A's role.

\section{FIGURE 1 NEAR HERE}

The answer to the first two requirements is that the cells should include more than one internal drives, which are independent "autonomous" components corresponding to those various cell roles. Each of these drives is endowed with an identification template, or identifier, that is compared against the corresponding templates of available messages to detect stimulation (template matching corresponds to message recognition). Messages have therefore two parts : an identification template part and an actual data part. The third proposition is true if cells are allowed to include multiple drives executing in parallel. In what follows, we will use the terms identifier and (eigen)frequency almost without any 
distinction. An important property of this model is that identifiers come for free, which implies that the connections' semantics may be dynamic. (NOTE 2)

To bypass the rigidity of usual symbolic messages as far as a comparison operation is concerned, we defined all the data and template parts to be real-valued numbers, and we introduced an extra noisetolerance in the case of template identification. We may adopt an analogy with physics to say that the identifiers recognized by the various drives are eigenfrequencies situated in a given scale (in the example that follows, it is the interval from -1 to 1 ), because among all possible frequencies only these ones can stimulate a cell. A cell choosing the interesting messages among all those available is a motivated cell.

To manage the drives' parallel activation and execution, we introduced an additional parameter of productivity or "speed" within each drive, which reflects the pace with which the drive scans the input messages. Actually, it is the speed of the reaction defined by the drive's transfer function. A drive having a high speed corresponds to a rapid "communication channel", in a way that its execution takes priority over the slower drives. A consequence of this scheme of "communication" in the network is that, in case of multi-party reactions, where there is need for fusion of messages coming from variable speed drives, the reaction depends on the latest message, i.e., on the slower drive of the previous activity level. This way, the total response speed of the cellular agent is the sum of all level speeds, where the speed of a given level is the minimum of the speeds of the drives belonging to this level.

Each drive has a minimum and a maximum speed and a rate of (gradual) change of speed (increase/decrease) according to presence or absence of excitation :

$$
\operatorname{speed}(t+1)=\operatorname{speed}(t)+s \_r a t e *\left(f i n a l \_s p e e d-\operatorname{speed}(t)\right)
$$

where final_speed is the maximum speed if the drive is stimulated (i.e., if there are messages that excite it) and the minimum speed otherwise. According to this simple adaptation law, the speed of each drive converges to the maximum or minimum speed, if it is constantly stimulated or not stimulated respectively, or fluctuates within these two limits in case of uneven and irregular stimulation. This way, a drive is self-catalyzed by its own activity, and the speed regulation mechanism follows an "eigendynamics" for each drive which is fast compared with the task's dynamics (that is, compared with the dynamics of the whole network's operation). In what follows, we will adopt a chemical analogy and we 
will refer to the drives' local speeds as reaction speeds. The reaction speed self-regulation mechanism is therefore responsible for the temporary differentiation of the cells with respect to the input messages. We also assume that each cell has a bounded and fixed maximal total productivity/speed, so that highly active drives tending to increase their local speed will compete with each other for cell resources (i.e., for fractions of the cell's total productivity). The cell's maximal productivity/speed corresponds therefore to an energy stock (such as a battery) from which all drives draw energy. This parameter is necessary in order to force competition between drives, otherwise a stimulated drive could self-catalyze without limit and there would be no competition possibility. Drives consume messages according to those internally expressed speeds, so that given enough genetic variation in initial speeds, the stronger or more rapid ones prevail by succeeding in consuming the relevant messages first, while the weaker ones gradually fade away. The "demands" for speed increase are processed in a manner proportional to the absolute current speed of the sender drives and are normalized according to the maximal cell speed. Overall, the "motivated" behavior of the cell emerges out of the competition of a set of independent "reactive" components, the drives.

Trying to make all the messages available to all cells, we may suppose at first glance an all-to-all connectivity between successive layers : this would lead to major bottleneck and inconsistency effects, since the same message would be processed independently by multiple cells and the drive competition mechanism would not function properly. To overtake this problem, we canceled altogether the connections between levels and defined instead shared message buffers where unique instances of messages output by cells of previous levels are put into. Alternatively, we may say that messages float in the cell interaction medium before being captured and processed.

Furthermore, those messages that would really need to be replicated for different cells (those that in the minimal algorithmic case stimulate more than one cells) have been defined as catalysts, i.e., messages that are not consumable by drives, but that are just "consulted" by them : actually, individual drives make local copies of the data part of catalyst messages. Moreover, and this corresponds to the chemical notion of catalysis, the consumable messages are ignored if the catalysts are not present. 
Finally, and to account for recovery from broader scale failures, we introduced an additional developmental/ontogenetical factor (NOTE 3), that slowly pushes unused drives' eigenfrequencies toward unused input message frequencies. In what follows, the adaptation point of reference is the closest identifier (frequency) :

eigenfrequency $(t+1)=$ eigenfrequency $(t)+e \_r a t e * c l o s e s t \_o f \_u n u s e d \_i n p u t \_f r e q u e n c i e s(t)$

The $e_{-}$rate parameter has to be very low compared with $s_{-}$rate, to avoid "alienation" of individual drives in case of non stimulation, i.e., to ensure a slow developmental shift compared with the drives' speed stabilization. This parameter will allow cells to self-organize by adapting "socially", i.e., to stabilize to frequencies present within the interaction medium (within the buffers) and thus coming from other parts in the network.

The robustness of the system to failures and to noise is mainly due to the presence of potentially large genetic variations in the variables that express eigenfrequencies, speeds and adaptation rates ("The real cause of stability in a distributed system is sufficient diversity”, Hogg \& Huberman (1993)).

The model is summarized in figure 2 .

FIGURE 2 NEAR HERE

\section{ILLUSTRATION : LEARNING TO NAVIGATE}

3.1 The Navigation Algorithm

We have shown in simulation the model's self-organizational potential for the case of the navigation system of an autonomous robot equipped with 4 obstacle and 4 trace sensors that heads toward a goal point by simultaneously following a line on the floor, if one exists. The robot's simulated world is a 2-dimensional grid, so that the simulated robot can only navigate in one of four possible directions (front, back, right, left). This way, each one of the four obstacle sensors and each one of the four trace sensors corresponds and is dedicated to one of the four navigation directions. Figure 3 depicts the robot in its navigation context.

The algorithmic control system is given in figure 4 and merges the information on the goal with the information on the obstacle and trace (line) sensors. We assume that the heading direction is fed by other parts of the network. In figure 4, the four leftmost "diff" cells compare the input direction with 
the output of the corresponding obstacle sensor and compute the deviation between the two or they give out a saturation value if an obstacle is perceived. The four rightmost "diff" cells do the same thing for the trace sensors. The cells of the next level sum the differences fed by the upper level; this sum expresses the total deviation of the corresponding direction from the desired heading and line direction. In this level, it will be necessary to eliminate first the directions blocked by obstacles and then favor those where a line is present. Finally, the decoder cell which is the motor driver chooses between the previous level messages the one proposing the minimum deviation. To this end, the different transfer functions are defined as follows :

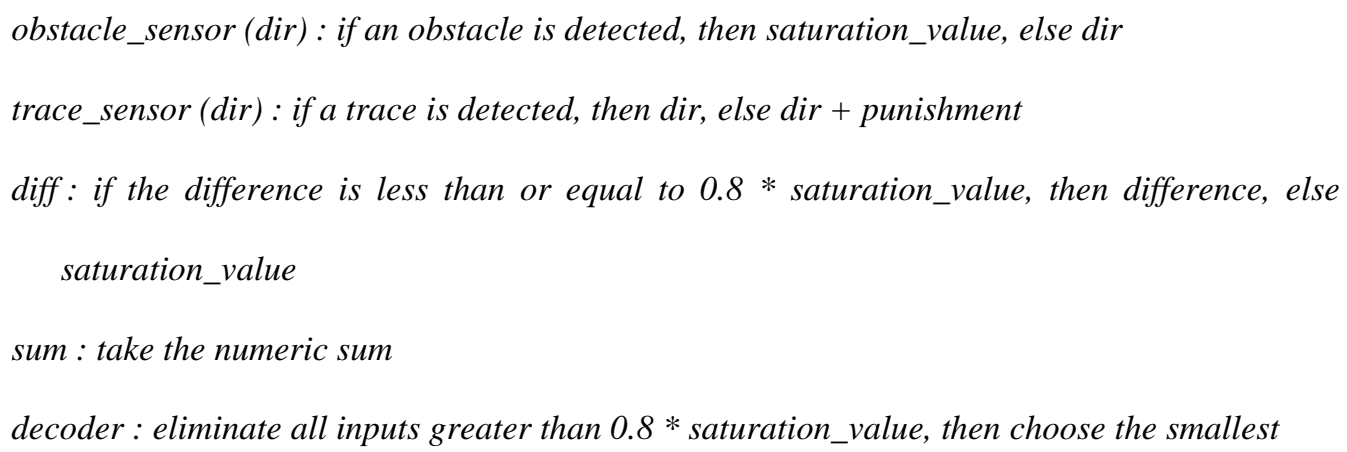

The obstacle sensor saturation value and the punishment value are 1000 and 5 respectively.

A former version of this navigation algorithm used a single trace sensor that was giving the best line direction (in order of preference, front, right/left or back) which was subsequently compared with the obstacle sensors output. This version has not been operational, because the agent, on arrival at the end of the line was doing a $180^{\circ}$ turn and was going back on its steps (Resnick \& Martin (1990) obtained the same result with a symmetrical line-following program on a Lego robot). To solve this problem, the trace sensor should be blind to the presence of a trace in the back, in which case the agent's trajectory would be in return suboptimal for a goal-point in the back. This problem revealed the need to compare the input (goal) direction with each one of the directions where a line could exist.

\section{FIGURE 3 NEAR HERE}

The messages traveling in the connections are real-valued numbers : directions take one out of four values $0.5,0.25,0.75,1$ corresponding to front, left, right and back, respectively. The saturation value is defined as 1000 , so that the messages arriving to the decoder and whose values surpass 1 may be eliminated. 
The corresponding self-organizing network is shown in figure 5. The sets of connections between the first three levels have been replaced by shared buffers, while the connections between the sum cells and the decoder have remained intact (because we did not introduce redundant decoder cells).

We have adopted as eigenfrequencies the same values used for direction discrimination : $0.5,0.25,0.75$ and 1 (that is, all drives processing the messages referring to the front direction have an eigenfrequency of 0.5 , and similarly for the remaining of the navigation directions). This scheme presents an advantage in our case : the heading sent to the motor by the decoder is the eigenfrequency of the chosen message, i.e., of the message corresponding to the minimum deviation (recall, however, that the frequency and the data part of a message are generally not correlated). The "diff" and the sum cells' drives need two identical input eigenfrequencies, so as to merge two incoming messages by taking the difference or the sum, respectively. To prevent obsolete messages from arriving to and being processed by the decoder, all the messages in the shared or private buffers are flushed every time the agent moves (this is equivalent to an additional "reset" input for the cell).

\section{FIGURE 4 NEAR HERE}

FIGURE 5 NEAR HERE (Both on the same page, please)

The goal direction message needs be shared among all diff cells, so it is defined as a catalyst. In the present navigation case, this means that the output of the obstacle and trace sensors is neglected if there is no goal direction given. The basic heading-by-line-following algorithm is designed for the case of static obstacles, that is, in an unknown but not hostile world. By construction, the algorithm is such that the directions (eigenfrequencies) about which no messages exist are ignored; if no messages about the left side make it to the decoder quickly enough, then no leftward movements will be undertaken, even if it is viable. In such a case, the agent will take bypasses or it will zigzag (for instance, an agent with the goal to go forward but not responsive to the front direction will make a zigzag to do so).

A final point concerns the representation space and its metrics. For a transfer function transforming signal A to signal B, A and B are points in a space and the transfer function represents an operation in this space. We are talking therefore about the action representation space, i.e., about the space in which the designer defines and designs the primitive cellular "actions" (or operations). This issue is discussed in depth in the third chapter of (Tzafestas 1995d). 
The problem with this navigation algorithm is that the space of the directions has to be a ring, so that the subtraction operation give, for instance, front-right=front-left, which is not possible with real numbers whose space is linear and unbounded (open). Moreover, this cyclic property has to be conserved during origin transposition, for instance $5+0.75$ has to be the same with $5+0.25$. Of course, I solved this problem by replacing the 0.75 differences by 0.25 , since only the values of $0,0.25$ and 0.5 are allowed and 0.75 corresponds to 0.25 if ones traverses the ring in the inverse direction. However, this is more a conceptual than a simple implementation problem : the form of the representation space should be coupled with that of the agent-world interaction. If the problem necessitates a ring configuration for the sensors, the primitive operations should be the operations of a ring. The solution is to define as representation space a mathematical space having all the necessary properties. It is certainly possible to define such a space on top of the real numbers space (as we did here), but, in view of a hardware implementation, it would probably be more practical to find a space having by definition all those desirable properties. Precisely, it looks to me that organic chemistry is a representation space extremely rich in properties such as symmetry, circularity and recursivity and chemical reactions are operations within this space that may become conveniently complex. In any case, the metrics and the topology of the representation spaces deserve the attention of artificial life researchers. What is the proper representation (mathematical) space best suited for a given problem and what are its minimal properties ? Up to now there has only been isolated and partial efforts : Thomas Ray (1991) used a mechanism for template addressing inspired by molecular biology and stressed the non euclidean nature of the computer geometry (Ray 1994), while Kephart (1994) insisted on the importance of topological structures for population dynamics.

\section{Sane Networks}

We simulated the operation of the navigation system for 3 to 5 cells in each of the two "diff" cell sets and in the sum cell set. Each cell was endowed with 1 to 3 drives with eigenfrequencies chosen among the four basic ones. Minimum and maximum speeds have been given random values in the ranges $(0$, $0.2)$ and $(0.8,1)$ respectively, whereas speed and eigenfrequency adaptation rates were given random values in the ranges $(0,1)$ and $(0,0.05)$ respectively. A typical trajectory for this agent is shown in figure 3. In all the experiments and keeping a constant sensor sampling rate, the network was found to stabilize quickly (in 10-20 cycles) to a configuration where 2 or 3 of the cells in every set were active, 
i.e., had drives working at speeds close to maximum, with the rest of the drives and cells being dormant, i.e., at minimum speed. The speed self-catalysis of the dominant cells leads to a self-catalysis of the total response speed, as figure 6 shows. (NOTE 4) Figure 7 gives the reaction speeds of the two drives belonging to the same diff cell : one can see that, as long as the network is stimulated, that is, as long as a goal heading is fed to the network, the stimulated drives are positively catalyzed and try to stabilize to their maximum speed, but as soon as the stimulation stops - here this happens because the goal is met and the heading cell outputs no message — the drives self-catalyze negatively and their reaction speeds fall back to minimum. The figure also shows the competition between drives that leads to a partial stabilization, that is to a speed configuration in which none of the two drives attains maximum speed, but the two drives find some sort of "compromise".

FIGURE 6 NEAR HERE

FIGURE 7 NEAR HERE

(Both on the same page, please)

3.3

Mild Lesions

Next, the following experiment was carried out: once the network had stabilized to a drive configuration (at $\mathrm{t}=100$ ), we removed one of the active diff cells (i.e., one of the diff cells containing active drives). Once more, it took the agent only a few cycles to re-stabilize to a new configuration ; actually, the self-organization process is by no means distinct from the normal network activity, so the network doesn't stop in order to learn the new configuration before going on. Instead, the navigation system continues operating as before, even if the direction affected by the lesion does not arrive to the decoder on time. Variation in minimum and maximum speeds of drives, as well as in their initial configuration, has as a result that the network does not always lose the direction affected by such a lesion, but often enough it remains sensitive to the direction at the price of sub-optimal response speed (figure 8a). Another relevant remark is that the cell physiology is such that, if suddenly an idle drive is stimulated, the active drives will find a speed compromise that will allow the re-stabilization of the global speed to a more operational value. So, for instance, in figure $8 \mathrm{~b}$, once a drive is stimulated, the second active drive of the same cell yields a fraction of its own speed to compromise with the first. 
FIGURE 8B NEAR HERE (both on the same page, please)

3.4

A Serious Wound

Next, we removed all the "diff" level drives that were excited by a given frequency (actually, all the relevant drives of one of the two diff cell sets). As before, the navigation system became irresponsive to the removed eigenfrequency, but this time it took around 50 cycles before a new message pathway was discovered, i.e., before one of the unused drives stabilized to that frequency (figure 9). This is of course an expected consequence of $e_{-}$rate being low, what necessitates a persistent stimulation of the network to ensure reconvergence to a stable configuration. Note also that the total speed of the agent decreased after the lesion, because the remaining cells had to redistribute their productivity among different drives. (NOTE 5)

\section{FIGURE 9 NEAR HERE}

"Developmental" adaptation, i.e., adaptation of drive eigenfrequencies, has to be continuous, that is the drive has to adapt its eigenfrequencies when it is stimulated as well as when it is not. The difference lies in the adaptation point of reference: when the drive is stimulated, it should adapt its eigenfrequencies according to the frequencies that have stimulated it, otherwise it should adapt its eigenfrequencies according to the closest input frequencies. Drives can then stabilize to persistent frequencies in a way that allows a co-stabilization of different level drives, that is in a way that allows the construction of a social standard or a common language in the network. An experimental demonstration of the need for continuous developmental adaptation may be found in (Tzafestas 1995b).

\section{5 \\ Discussion of the Model and the Results}

The introduced cell model is an extension of the basic model of section 2 and differs from traditional neuron models in two fundamental ways :

- There are no connections between cells, but cells "float" in a common medium and share message buffers with other cells. This allows and simultaneously necessitates a competition between cells for the consumption of available input messages. 
- Cells respond to messages at their own pace and according to their local preferences/motivations. They are not simply "input machines" and therefore manipulable machines, but they possess a degree of individual autonomy that shows as selectivity toward the input messages. Selectivity is coupled with a reaction speed regulation mechanism, i.e., with a mechanism that modifies the dynamics of interaction with the world.

Just like connectionist models, though, cells adapt continuously and in parallel with activity, i.e., the adaptation and action phases are not separate (not only does the agent adapt without a supervisor, but it has no idea that it adapts). The memory of the system is medium term, and so it does not present the usual forgetting and short memory problems. If it needs to forget, it will! This adaptation process is based on purely endogenous parameters and does not depend on a benevolent teacher that would have to prepare the learning data, choose the language and the abstraction level of representations (van de Welde (1991) insisted on the need to carefully rethink those questions). According to Kaelbling's (1991) criteria, such a cellular network is ensured to converge to a correct configuration using an adaptation mode of constant spatio-temporal complexity. Let us not forget, however, that the agent does not learn anything new on its environment, but only how to use best its innate potential ; a scaling study to topological or other learning problems remains to be done.

This model gives room to the introduction of genetic variations and to the emergence of division of labor between cells that arises. Furthermore, such a network of motivated cells remains largely understandable and in the case of major failures an engineer may intervene directly to the drive level and upgrade the system manually, since the algorithmic character of activity/message processing is preserved. In this sense, variation allows self-organization that ensures qualitative stability, or algorithmic operationality. Besides, the continuous nature of data and frequencies will allow a future dynamical analysis of these networks. The adaptation rates as well as the maximum/minimum speeds may also be defined as variable throughout the agent's life ; I can imagine decreasing adaptation rates that will make the agent decreasingly robust to failures and decreasing speed limits that will make the agent decreasingly productive (cf. Tzafestas 1996).

The temporal behavior of the network is such that it is ensured to converge to a stable configuration if continuously stimulated and in any case it will "follow" any "capricious" stimulation regimes, according to its internal motivations. The self-catalytic cellular behavior makes the agent appear lazy (work at slow speed) in the absence of important perturbations, but increase productivity drastically 
when stimulated. This self-regulatory behavior of the cell demonstrates the significance of time as a network design parameter (issue raised and analyzed in the dynamical neural networks literature, for instance Husbands et al. (1993), Yamauchi \& Beer (1994)). As long as the network operates near random initial conditions or for a while after the occurrence of lesions, the behavior of the agent appears as non-coordinated and suboptimal, for instance the agent might wander a little where one would expect it to go straight ahead.

Selectivity. Having motivated cells, i.e., cells that decide whether they will process a message or not and what they are going to do with it, implies that cells are no more directly instructed by their environment in which they are situated, but rather that they are selective toward it; it is no more information transmitted from the world to the agent, but personalized perturbation of the agent by a subset of environmental properties. As figure 10 shows, interactions are no longer instructions sent from the world to the cells, but they are instead true acts of selection by the cells. This way, plasticity becomes an essentially nodal, rather than a synaptic, property. Let us point out, however, that selectionist instead of instructionist modeling does by no means change the form and the nature of emergent phenomena. From a design viewpoint, it may simplify the task of programming/construction of control systems that are simultaneously algorithmic and plastic, and it also appears promising for a more causal exploration of these same phenomena (Varela 1979). The importance of selection systems for the future of cognitive science has been stressed by Edelman (1992) who analyzes the recognition sciences (evolution, immunology and neurobiology), i.e., the sciences of the recognition systems, that are systems having a diversity generator, a heredity mechanism and a mechanism of amplification of selective events. We will come back to the notion of selection in section 5.3.

FIGURE 10 NEAR HERE

We have seen that the developmental adaptation mechanism allows the network to find a stimulation pathway even in the case of major failures in the network. Actually, cells adapt to the frequencies of the input messages without deciding on the legality of those frequencies. This may lead to a network alienation if those messages are more or less false, for instance if those messages' frequencies are subject to "mutations". If mutations persist, drives will stabilize to frequencies detrimental for the 
network's integrity. What do we do ? The solution is to equip the network with a possibility to distinguish between valid and invalid messages, that is to introduce an "immune system" :

- The alien frequencies should be detected and the carrying messages eliminated.

It is easy to imagine that this situation corresponds to a cooperation game : the "intruder" frequencies ought to be recognized as different from the legal ones — therefore as detrimental — to be eliminated. This observation leads us to a view of the cell's social behavior as a behavior based on similarity measures (genetic kinship, in terms of theory of evolution language). To this end, another type of cells has been introduced, the defender cells, entitled to recognize and eliminate intruders. Note, however, that the initial idea of defenders recognizing the good eigenfrequencies and eliminating those with low affinity has been found faulty: in that case, and given that more than one eigenfrequencies are supported by the network, a frequency considered as an intruder by a certain defender might be perfectly valid from the viewpoint of another one and the network would then be instable. The alternative is to have defenders that recognize directly the bad frequencies to eliminate : the defender cells would therefore have eigenfrequencies values known to be bad, i.e., eigenfrequencies complementary to the valid ones. This formulation of the recognition problem has two important features :

- The cellular model remains selective. Defender cells follow the same cellular model as the producer cells, with the only functional difference that they "swallow" the messages that excite them without metabolizing them.

- The network comprises therefore two complementary social forces, the "production" force, that is responsible for the completion of the agent's task, and the "supervision" or "police" force, that is responsible for the detection and the elimination of the alien messages for the same task.

To model this social cellular behavior, we used the quantitative tit-for-tat model (Tzafestas 1995e) according to which a "social" stimulus is only considered cooperative when its value is superior to a certain threshold ( $f($ stimulus $) \geq T \Rightarrow$ cooperation, else defection). This model allows the description of a whole class of social relations — participation as well as game relations - with variations in the parameters (such as the threshold $T$ ) as well as in the structures (such as the perception and cooperation functions).

The social behavior of the control system cells may be described in terms of this model as follows : 
- perception function (or social stimulus) $f\left(x_{1}, \ldots, x_{n}\right)=\min \left|x_{i}-e\right|$, where $x_{i}$ are the frequencies of the input messages and $e$ is the eigenfrequency of the drive (actually, the social adaptation affects all the eigenfrequencies of a drive),

- cooperation function : developmental adaptation, that is eigenfrequencies shift, plus execution of the transfer function in case of stimulation, like in the motivated model (the transfer function is a specific function for each drive belonging to a producer cell and a swallowing function for each drive belonging to a defender cell),

- defection function : do nothing for both types of cells.

The final type of studied failures is alien messages that are not forced into the buffers by some external agent but are produced by capricious, malevolent or simply faulty cells. In that case, eliminating the messages is not sufficient ; on top of that, we need a way to prevent those cells from taking control of incoming messages and metabolize them abnormally.

- The network should have self-correcting potential, i.e., the malignant cells should converge back to a proper behavior.

To this end, we added the possibility of internal drive adaptation (adaptation/ convergence of the output frequency toward the input frequency) according to a social participation criterion :

$$
\text { participation }<0 \Rightarrow \text { internal adaptation }
$$

where participation $=f_{\text {output }}-f_{\text {input }}, f_{\text {input }}=1$ if the drive is stimulated and 0 otherwise, $f_{\text {output }}$ is the proportion of output frequencies corresponding to messages present in the output buffers (actually, the mean of proportions concerning all the output buffers). The inequality holds if one or more output messages are consumed by the defender cells.

The social cell behavior and the social network configuration may be summarized as follows :

- There are two parallel cellular systems : the producer cells and the defender cells whose excitation frequencies are complementary (NOTE 6). The frequencies recognized by the defenders are detrimental for the social network's integrity. The defenders' transfer function is a function of swallowing of the stimulating messages (no message output). 
- The social behavior of all drives follows a quantitative tit-for-tat model : the cooperative behavior is developmental adaptation, while the non-cooperative one is to do nothing (no social participation). All drives compute the messages' affinity to their own eigenfrequencies according to a monodimensional distance criterion.

- There is an additional measure of social participation of drives that stimulates an internal adaptation process : if participation is negative (i.e., if messages "produced" by the drive disappear quickly thanks to defenders intervention), the adaptation affects the output rather than the input frequencies.

- Once more, the power of the social cell model lies in its potential for large variations in parameters (thresholds, rates, frequencies etc.) as well as in structures (metabolic transfer functions and adaptation variants).

The issue of the population. The participation measure used is "binary" : if the drive participates normally to the process, then this measure will be 0 , otherwise it will be inferior to 0 , in which case "internal" adaptation will take place. Another possibility considered in perspective is to endow the cells with an additional speed regulation mechanism according to a measure of continuous participation depending on the proportions of input and output frequencies in the corresponding buffers. In this case, we will be able to talk about regulation by social feedback that will show as "division of labor" and that will become necessary in case of large populations with redundancy at all levels : as far as stability is concerned, an algorithmic solution — as well as a minimal solution - will suffer from occasional centralization (as is for example the case of the decoder cell) and could therefore not be able to profit from diversity which is the basis of stability in distributed systems. A diversity generator in combination with the cooperation game between the cells of the two types may give rise to phenomena that might be viewed as learnt behaviors. An additional study that I intend to carry out concerns the dynamics of a producer-immune control network especially designed for a problem of navigation with spatial learning: what kind of dynamics does it emerge? And what are the necessary types of dynamics that may allow for such learning ? (NOTE 7)

The limits. For the sensor and actuator cells that do not have input and output frequencies (and that are therefore situated in the frontiers of the agent with its world), it does not make sense to speak about developmental adaptation (in the implementation, the developmental adaptation factors of those cells' drives have been fixed to 0 ). The interaction modes with the world are fixed and correspond to the 
"physical" perception and actuation abilities : a mammal could decide to fly, but it could never fly. Those limits are therefore the cell's adaptation limits and by extension the learning limits of the cellular agent. I imagine that an artificial nervous system coupled with an artificial immune system would demonstrate the same inferiority in terms of adaptation possibilities. I also imagine that it would be interesting to introduce completely internal boundary "nervous" parts, i.e., neither perception nor actuation nervous systems, that would determine fixed modes of esoteric interactions.

The world of representations. It is interesting to evoke once more the problem of the representation space : a diversity generator would have to provoke "mutations" whose gradual accumulation could take the form of the messages far away from their initial point. However, those mutations should not be too abrupt, otherwise the semantics of interaction between cells could collapse and the network could disintegrate. Resistance to "noise" is therefore necessary. But mutation and resistance to noise are not everything : it would be preferable to have multiple alternative trajectories between two points in this space, in which case the diversity generator would produce a range of mutated forms that would explore the space of the possibilities in parallel. The gain would not lie in the parallelism, though, but in the existence of multiple alternatives, hence of redundancy : in the space of real numbers there is unfortunately only one possibility to go from 0.3 to 0.5 , the metrics of this space is linear. Once more, organic chemistry appears to me the perfect space for such a diversity.(NOTE 8)

We have carried out a first experiment to show that even with a random intrusion there may be alienation of the network in the sense that certain drives stabilize to useless frequencies and one or more navigation directions (that are essential for the navigation task) disappear from the network. Random intrusion means that with a certain probability (the intrusion rate) the frequency of a message of the concerned buffer is mutated randomly; the new frequency may therefore be valid or not for the network, but its generation remains stochastic. The results of a simulation with an intrusion rate of 0.4 in the input buffer of one of the two diff levels are given in figures $11 \mathrm{a}$ and $11 \mathrm{~b}$. We can see that, 100 cycles later, the network is no more responsive to the right direction, since the drive that was responsible for this direction has been alienated. Generally, as a number of experiments have shown, a 
high intrusion rate results in developmental disintegration, such that there is a diversity of non correlated frequencies, i.e., there is no meaning for these frequencies.

\section{FIGURE 11A NEAR HERE}

\section{FIGURE 11B NEAR HERE}

We repeated the same experiment with the additional possibility of intrusion of virus, that are messages having an alien data part as well as an alien frequency part (an alien data part is a data part randomly mutated). As simulations have shown, this time not only is the network alienated and certain frequencies disappear from it, but also, since there may be messages with a good frequency and a bad data part, a second type of alienation exists, a more semantic alienation that causes collisions of the agent with the walls and the obstacles.

We have therefore added around each of the buffers two to four defender cells having one to four drives with eigenfrequencies taking one of the following values : $0.125,0.375,0.625$ or 0.875 . Those frequencies are situated in the middle of the controlled intervals, i.e., the middle of the intervals between successive operational frequencies. The cooperation detection thresholds of the defenders' drives have been defined in the same way, i.e., as approximately half of the controlled intervals, around 0.06. The intrusion of mutated messages or virus as before continued to cause partial destabilization of the network in the sense of occasional loss of one or more navigation directions, but did not lead to network alienation. In any case, to ensure the network's operationality, the defenders' drives ought to have high minimum speeds and their number should be sufficiently high. (NOTE 9)

\subsection{Malignant Cells and Auto-Immune Response}

Finally, we experimented with faulty producer cells whose output messages were distorted: their frequencies as well as their data parts were taking random values (always between 0 and 1). In any case and given enough defender cells, the messages carrying alien frequencies have been correctly eliminated. However, for the messages encapsulating a good frequency with a bad data part, results have been subtler: even if alienation of the network has not been possible, there are zigzags and bypasses and this up until the faulty cell discovers the proper pathway (often, the agent may no more be able to attain its goal-point). 
This experiment shows that the sole existence of a passive "social" restabilization mechanism of malignant cells is not sufficient, and the reason is twofold :

- It is possible that the malignant cells have only a limited restabilization possibility (or even none!). This is the case of very low developmental rates (close to 0 ).

- The consequences of the loss of sensitivity to a given message type for such a long period may be fatal.

What is necessary is the possibility to recognize not only the bad message, but also the source of this message. This way, an isolated message, be it an intruder or a virus, will simply be eliminated. On the contrary, for a message coming from another cell in the network, this cell has to be recognized and "punished" in a way or another. Note that the "social" stabilization mechanism relies on such a "punishment" measure (the absence of participation to the activity of the social network). However, such "distributed" punishment is not enough : the social network should include an authority that will be charged with punishment. In the case of faulty cells, the authority, i.e., the immune network, should either intervene directly in the cells' program or simply kill them, in any case show direct aggressiveness. The first option would necessitate a rather complex intervention program, while the second appears to be a simple solution in the case of cells that cannot proliferate.

The adaptive and self-organizational abilities of immune systems have stimulated research in optimization and adaptive control (Bersini \& Varela 1991, Bersini 1992). On the other hand, the idea to apply principles borrowed from immunology to robotics is not new. The biological adaptation systems in general (including the immune system) have been vaguely proposed as an inspiration source for the development of process control systems (Renders \& Hanus 1992). The immune system paradigm is used in the action selection mechanism of (Ishiguro et al. 1995), which is a direct descendant of Maes' mechanism (1991). Our mechanism has been developed with the aim to reveal principles of cellular organization rather than to provide solutions to engineering problems; the navigation problem has only served to illustrate these principles.

\section{3}

\section{General Discussion}

We have exposed the philosophy behind plasticity and self-organization in an algorithmic cellular network. We have shown that, in order to allow for this kind of plasticity, it has been necessary to do 
two major extensions to the original cell model : (a) introduction of local motivations/preferences in the cells, that are expressed in an uniform manner as excitation eigenfrequencies and that are compared to the identifiers of the input messages, and (b) replacement of the connections between levels by shared message buffers. Another extension consists in defining two message types, the usual consumable messages and the catalytic messages whose sole presence is enough to trigger the consumption of other messages by the excited cells; all messages have two parts, an identification and a data part. Some simulation results reveal that the cellular network self-organizes in case of failures by discovering alternative message pathways and that multiple failures slow down the system's reactivity to external events, since the cells of intermediate levels are no more specialized to one message type, but share their time between multiple motivations. Furthermore, the presence of an additional developmental/ontogenetical factor allows for self-organization even in the case of major failures in the network, i.e., when an eigenfrequency disappears completely from the network.

Note that the social behavior of the motivated cell is by definition "cooperative", i.e., the cell will try to stabilize to a new frequency, even if this frequency is detrimental for the network's integrity. What is necessary in this case, is an immune system, i.e., a cellular system parallel to the first, supervising and regulating the "social" adaptation of the other cells. These cells are motivated, too (they also ought to be social, but in the studied example their sociality has not been used) and their excitation eigenfrequencies are complementary to those of the producer cells, what has as a consequence that recognition and elimination of enemy-messages is straightforward. By default, the social behavior of all cells is cooperative, regardless of their type : the integrity of the network is therefore preserved thanks to the coupling of two social forces, the production and the police. A study of the integrity of the network is therefore translated into a study in the meta level, the level of the matching adaptive motivations network, i.e., the network of the cells' eigenfrequencies. Note also that there is no need for a meta-immune system, since, in principle, the immune system is allowed to act upon itself provided that defender cells are sufficiently numerous and diverse. Pitrat (1991) insists that all knowledge is situated at a meta level and that one single level of meta-knowledge with the possibility to act upon itself, that is with reflexivity, suffices for the development of cognitive abilities comparable to those of humans and even greater.

For the set of the two systems (the production and the immune system) to be coherent and nonmanipulable, the population of frequencies has to be developed by the agent itself during its life as a 
result of interactions in the network. The most important perspective of the presented work is the study of the co-development of the two networks in the abstract level of the eigenfrequencies representation space, i.e., in the level of information (be it unidirectional, like the real numbers space, or not) : I am convinced that a "learnt" behavior is in reality a "developed" behavior.

From selectivity to selectionism. If the cells/drives are selective, a learnt behavior will in fact be a behavior generated by the network itself and reinforced in a particular environment. Selectivity has to be combined with a diversity generator and an environmental amplification (or reinforcement) mechanism to allow for apparent learning. In the case of the autonomous cell model, it would be necessary to introduce a mechanism for eigenfrequency generation (or revision). There would then be a tug-of-war game between the two cell populations, in which the ones would try to generate new frequencies and the others to eliminate them; in this case, the recognition and elimination of alien eigenfrequencies would be just a side-effect of the cells' social game. And the question would become : in a stable environment, does the network stabilize to a configuration? Does it "learn" something? Manderick (1992) analyzes and compares three selective systems (natural evolution, the immune system and the brain) stressing the importance of selectionism for the design of autonomous cognitive systems in the context of the nouvelle (or behavior-based) AI. (Steels 1996) remains so far the only example of variation and selection inside the control system of a robot and is inspired by the selectionist brain theories developed by Edelman.

\section{6}

\section{CONCLUSION}

This article presented the problem of a cellular organization of dynamic fault-tolerant topology. A network organization is cellular when the components have heterogeneous transfer functions, but the connections syntax is homogeneous. The basic input-output cellular model is extended to a selective and motivated model of autonomous cell. The extended model relies on the definition of the cell as a system of components, the drives, executing in parallel and being in constant competition with one another for the consumption of the messages received by the cell. While individual drives are responsible for the messages consumption, a speed regulation mechanism is responsible for the coordination and the conflict resolution between drives. In order to work, the mechanism necessitates compound messages, that comprise an identification and a data part. Some of these messages ought to 
be catalysts that will trigger the reactions of other cells without being literally consumed. The operationality of the model and its robustness to various types of failures are ensured by the speed regulation mechanism that modifies the dynamics of the interaction of the drives with the world of the messages; this modification concerns the parameters that determine the dynamics of agent-world interaction (here, message consumption, or reaction, speeds) and is homeostatic. Sociality shows as the adaptation of the cell to the form of the encountered messages.

The most spectacular consequence of the model is, however, the need for an immune system. In the case of alien messages and viruses that may alienate the network, and make it give false answers to its perturbations, a cellular defense system parallel to the first one, i.e., an immune system, appears necessary. The motivated cell model is therefore further modified to include the functionality necessary for an elementary immune system : two types of cells are defined, the "producer" cells and the defender cells. The defense system relies on the recognition and elimination of alien messages by defender cells. The coupling between the defense and the production system shows in the complementarity of the messages recognized by the two systems, it is hence a coupling between two social forces within the same cellular agent. The problem of the representation space is also evoked and briefly discussed in a future learning perspective.

The essence of the cell's autonomy is the continuous change, the continuous modification or adaptation of the dynamics of interaction with the world (cf. van Gelder \& Port (1995)). Change or adaptation goes hand in hand with the agent's selectivity, and its purpose is to make the agent increasingly selective to the relevant aspects of the environment and decreasingly selective to the irrelevant ones. Adaptation is in fact a self-regulation of the parameters determining this dynamics, hence the self-regulation of parameters having a temporal character, rates or speeds. Concerning the relation between autonomy and self-regulation of adaptation rates, the same result has been obtained at the cellular agent level (chapters 4 and 5 of (Tzafestas 1995d) and (Tzafestas in prep.)) and supports our claim for recursive organizational principles, i.e., for organizational principles applicable at all agent levels. Of course, adaptation possibilities are by no means limited to parameter regulation and further alternatives are considered in perspective — in particular the developmental approach, especially when coupled with an immune control system, as discussed in section 5.3. 
In the case of the cellular self-organization mechanism, to ensure continuous change and learning, it could be useful to have a mechanism inducing failures and thus injecting noise continuously (for instance a senescence function, such as the one developed in Tzafestas (1996)).

Finally, we stressed the role of diversity and self-organization inside the agent; besides the pure operationality considerations presented above, we have sketched the relation of diversity with selectionism and the perspectives that open up in the direction of developmental learning.

\section{TABLE 1 NEAR HERE}

\section{ACKNOWLEDGMENT}

I wish to thank Philippe Darche for fruitful discussions.

\section{REFERENCES}

Almássy, N., Verschure, P. 1992. Optimizing self-organizing control architectures with genetic algorithms: The interaction between natural selection and ontogenesis, Technical Report No. 92.10, Department of Computer Science, University Zurich-Irchel, 10p, also in: Proceedings of the Second Conference on Parallel Problem Solving from Nature (PPSN '92), edited by Männer, R. \& Manderick, B., North-Holland.

Arkin, R. 1987. Motor schema based navigation for a mobile robot : An approach to programming by behaviour, Proceedings 1987 International Conference on Robotics and Automation, Raleigh, NC.

Arkin, R.C. 1989. Motor-schema based mobile robot navigation, International Journal of Robotics Research, 8, 92-112.

Beer, R. 1990. Intelligence as adaptive behavior - An experiment in computational neuroethology, San Diego, CA, Academic Press.

Beer, R.D., H.J. Chiel, L.S. Sterling (1989). Heterogeneous neural networks for adaptive behaviour in dynamic environments, in: Advances in Neural Information Processing Systems 1, by D.S. Touretzky (Ed.), Morgan Kaufmann, 577-585. 
Beer, R.D., H.J. Chiel (1991). The Neural Basis of Behavioral Choice in an Artificial Insect, in: From Animals to Animats, Proceedings of the First International Conference on Simulation of Adaptive Behavior, edited by Meyer, J.-A. \& Wilson, S.W., Cambridge, MA, Bradford/MIT Press, 247-254.

Bersini, H., Varela, F.J. 1991. The immune recruitment mechanism: A selective evolutionary strategy, Proceedings of the 1991 International Conference on Genetic Algorithms.

Bersini, H. 1992. Immune network and adaptive control, in: Toward a practice of autonomous systems, Proceedings of the First European Conference on Artificial Life, edited by Varela, F.J. \& Bourgine, P., Cambridge, MA, MIT Press/Bradford Books, 217-226.

Brooks, R.A. 1986. A robust layered control system for a mobile robot, IEEE Journal of Robotics and Automation, 2, 14-23.

Brooks, R.A. 1990. The Behavior Language - User's Guide, M.I.T. AI Memo No. 1227, 35 p.

Brooks, R.A. 1991a. Intelligence without Representation, Artificial Intelligence, Special Issue on the Foundations of Artificial Intelligence, 47, 139-159.

Brooks, R.A. 1991b. Elephants don't play chess, in: Designing Autonomous Agents : Theory and Practice from Biology to Engineering and Back, edited by Maes, P., Cambridge, MA, MIT Bradford Press, reprinted from Special Issue of the Journal on Robotics and Autonomous Systems, 6, June 1990, 3-15.

Cangelosi, A., Parisi, D., Nolfi, S. 1994. Cell division and migration in neural networks, Network, 5, 497-515.

Chapman, D. 1990. Vision, Instruction and Action, Ph.D. Thesis, M.I.T. Artificial Intelligence Laboratory Technical Report TR-1204, April 1990.

Cliff, D., Harvey, I., Husbands, P. 1993. Explorations in evolutionary robotics, Adaptive Behavior, 2, 73-110.

Edelman, G.M. 1992. Bright air, brilliant fire: On the matter of mind, Basic Books, 1992.

Elman, J.L. 1993. Learning and development in neural networks : The importance of starting small, Cognition, 48, 71-98. 
Ferrell, C. 1994. Failure recognition and fault tolerance of an autonomous robot, Adaptive Behavior, 2, 375-398.

Gruau, F. 1995. Automatic definition of modular neural networks, Adaptive Behavior, 3, 151-183.

Hogg, T., Huberman, B.A. 1993. Better than the best: The power of cooperation, in: SFI 1992 Lecture Notes in Complex Systems, edited by Nadel, L. and Stein, D., Addison-Wesley, 163-184.

Husbands, P., Harvey, I., Cliff, D. 1993. Analysing recurrent dynamical networks evolved for robot control, Cognitive Science Research Paper 265, Univ. of Sussex.

Ishiguro, A., Watanabe, Y., Uchikawa, Y. 1995. An immunological approach to dynamic behavior control for autonomous mobile robots, Proceedings IROS'95, Pittsburgh, PA.

Kaelbling, L.P. 1991. Foundations of learning in autonomous agents, Robotics and Autonomous Systems, 8, 131-144.

Kephart, J.O. 1994. How topology affects population dynamics, in: Artificial Life III, edited by Langton, C.G., Reading, MA, Addison-Wesley, 447-463.

Maes, P. 1991. A Bottom-Up Mechanism for Action Selection in an Artificial Creature, in: From Animals to Animats, Proceedings of the First International Conference on Simulation of Adaptive Behavior, edited by Meyer, J.-A. \& Wilson, S.W., Cambridge, MA, Bradford/MIT Press, 238-246.

Manderick, B. 1992. Selectionist systems as cognitive systems, in: Toward a practice of autonomous systems, Proceedings of the First European Conference on Artificial Life, edited by Varela, F.J. \& Bourgine, P., Cambridge, MA, MIT Press/Bradford Books, 441-447.

Pitrat, J. 1991. Métaconnaissance - Futur de l’Intelligence Artificielle, Paris, Hermès.

Ray, T.S. 1991. An approach to the synthesis of life, in: Artificial Life II, Proceedings of the Workshop on Artificial Life, edited by Langton, C.G., Taylor, C., Farmer, J.D. \& Rasmussen, S., Addison-Wesley, 371-408.

Ray, T.S. 1994. An evolutionary approach to synthetic biology: Zen and the art of creating life, Artificial Life, 1, 179-209. 
Renders, J.-M., Hanus, R. 1992. Biological learning metaphors for adaptive process control : A general strategy, Proceedings of the 1992 International Symposium on Intelligent Control, Scotland.

Resnick, M., Martin, F. 1990. Children and Artificial Life, M.I.T. E\&L Memo No. 10,13p.

Steels, L. (1994a). The artificial life roots of artificial intelligence, Artificial Life, 1, 75-110.

Steels, L. (1994b). Emergent functionality of robot behavior through on-line evolution, in: Artificial Life IV, Proceedings of the Fourth Interdisciplinary Workshop on the Synthesis and Simulation of Living Systems, edited by Brooks, R. \& P. Maes, MIT Press, Cambridge, MA, 8-14.

Steels, L. 1996. Discovering the competitors, Adaptive Behavior, 4, 173-199.

Stewart, J., Varela, F.J. 1991. Morphogenesis in shape-space, elementary meta-dynamics in a model of the immune metwork, Journal of Theoretical Biology, 153, 477-498.

Tzafestas, E.S. 1995a. Variation et auto-organisation à l'intérieur du robot: Le cas d'un modèle de fréquences propres, Actes des Journées de Rochebrune “Évolution et organisation”, Rochebrune, France, 181-192.

Tzafestas, E.S. 1995b. Compromising algorithmicity and plasticity in autonomous agent control systems: The motivated, social cell, Accepted for a poster presentation 1995 European Conference on Artificial Life (ECAL'95), Granada, Spain. Extended version: LAFORIA Research Report 95/33, 35p.

Tzafestas, E.S. 1995c. Peripheral cellular control: An eigen-frequency model and a case-study in selforganization, Vol. I, Proceedings 1995 IEEE/RSJ International Conference on Intelligent Robots and Systems (IROS'95), Pittsburgh, PA, 483-488.

Tzafestas, E.S. 1995d. Vers une systémique des agents autonomes : Des cellules, des motivations et des perturbations, Thèse de Doctorat de l'Université Pierre et Marie Curie, Paris, December 1995.

Tzafestas, E.S. 1995e. Beyond cooperation and competition : Explorations with a quantitative tit-for-tat model, LAFORIA Research Report 95/34, 23p.

Tzafestas, E.S. 1996. Aging agents, LAFORIA Research Report 96/01, 22p.

Tzafestas, E.S. in prep. Regulation Problems in Explorer Agents, in preparation.

van de Welde, W. 1991. Toward learning robots, Robotics and Autonomous Systems, 8, 1-6. 
van Gelder, T., Port, R. 1995. It's about time: An overview of the dynamical approach to cognition, Research Report 116, Indiana University at Bloomington, Dept. of Cognitive Science, October 1994, 39p., also in Mind as Motion : Explorations in the dynamics of cognition, edited by Port, R. \& van Gelder, T., Cambridge, MA, Bradford/MIT Press.

Varela, F. 1979. Principles of biological autonomy, Elsevier/North-Holland, New York, French edition “Autonomie et connaissance : Essai sur le vivant”, Paris, Éditions du Seuil.

Verschure, P.M.F.J., Kröse, B.J.A., Pfeifer, R. 1992. Distributed adaptive control : The selforganization of structured behavior, Robotics and Autonomous Systems, 9, 181-196.

Verschure, P.M.F.J., Pfeifer, R.. 1992. Categorization, representations, and the dynamics of systemenvironment interaction: A case study in autonomous systems, in From Animals to Animats 2, Proceedings of the Second International Conference on Simulation of Adaptive Behavior, edited by Meyer, J.-A., Roitblat, H.L. \& Wilson, S.W., Bradford/MIT Press, 210-217.

Yamauchi, B.M., Beer, R.D. 1994. Sequential behavior and learning in evolved dynamical neural networks, Adaptive Behavior, 2, 219-246. 


\section{NOTES}

(*) Part of this work has been completed while the author was a Ph.D. candidate in the LAFORIA-IBP (Laboratoire Formes et Intelligence Artificielle, Institut Blaise Pascal, Université Pierre et Marie Curie, Paris, France) under the supervision of Professor Jacques Ferber. Earlier or partial versions of this article appeared in (Tzafestas 1995a,1995b,1995c).

(1) Ferrell (1993) implemented on the hexapod robot Hannibal an ad hoc algorithm of failure recovery relying on a hierarchical decomposition of the fault detection process and on a clear distinction between hardware (sensors/actuators) and software (program compiled onto the processors). I am exploring here the idea of failures in a network where such a distinction is absent : everything is of the same nature, everything is a cell, and especially the "software" is a network of "material cells", too!

(2) In the example that follows, all the input/output frequencies of a drive are identical. This is not but a specificity of the example; in principle, the cellular model does not impose any such constraint.

(3) I use the term "development" in the same sense as Verschure \& Pfeifer (1992), Almássy \& Verschure (1992), to show that, if the environment is stable, i.e., if it does not change qualitatively, the network will gradually stabilize to a permanent operational configuration without growing.

(4) This total speed is computed as the sum of the reaction speeds of different levels, where the reaction speed of a given level is actually the lowest speed of all the reactions that take place (recall that multi-party reactions represent convergence or fusion points in the network, cf. section 2.1).

(5) I tried to elaborate a stabilization model that would remind what happens in the living organisms, such as animals, where, after recovery from nervous system lesions, the performance of the system is lower as far as the response speed is concerned (my lesion study resembles the one carried out by Beer (1990)).

(6) Note however that, as defined, the defender cells do not constitute a system in the true sense of the term (there are no relations between them). In an extension of the model, it will be 
necessary to define a true defender cell network parallel and structurally homomorphic to the production network.

(7) Stewart et Varela (1991) stress and analyze the importance of two coupled dynamics, the "reaction" dynamics and the meta-dynamics that generate and select new reactions. In our model, this would induce a need to include a possibility of developmental "revision" of eigenfrequencies.

(8) I would like to know which is the minimal "molecular" level that we need. I tend to think that messages would be more resistant to noise, if they were systems, too : a string of symbols, such as abbcabxa or 10001101101, is not a system because there is no relation between the different symbols and each one may mutate independently of its neighbors — but this is not the case of proteins.

(9) Since defender cells should not proliferate, their number should be high enough from the beginning. A good estimate to take is the size of the controlled buffer. 


\section{FIGURE LEGENDS}

Figure 1 A 3-layer cellular structure

Figure 2 The drive model

Figure 3 The agent in its navigation universe. Line following with simultaneous obstacle avoidance. The dark parts of the "surface" stand for the traces and the small grids stand for the obstacles. The dot line is the agent's trajectory. The deviations of the agent from the "optimal" trajectory are due to the presence of a degree of noise (here $10 \%$ ) in the generation of the line direction, what has as a consequence that the chosen direction is not always locally optimal ; this noise allows to the agent to avoid getting trapped in dead-ends.

Figure 4 The algorithmic navigation system

Figure 5 The self-organizing navigation system

Figure 6 Total response speed stabilization. This speed is actually the total reaction speed of the agent, i.e., a measure internal to the agent, and does not necessarily represent a true speed as perceived by an external observer (cf. section 2.1).

Figure 7 Drive speeds competition for a "diff" cell. In the first part of the curve the drives are stimulated, so their speeds rise toward their maximum ; in the second part, the drives are no longer stimulated and their speeds fall to their minimum. Note that the highest speeds of the two drives are not their maximal ones, they are away from 1.

Figure 8a Re-stabilization of total speed. At $t=100$ removal of a diff cell that used to process the right direction $(0.25)$. The total speed decreases after the lesion and re-stabilizes later $(\mathrm{t}=200)$.

Figure 8b For the experiment of figure 8a, speed re-stabilization of two drives after the lesion $(\mathrm{t}=100)$ and the recovery.

Figure 9 The response direction (or response identifier) of the drive that undertook the role of "front".

Figure $10 \quad$ (Left) Instructive model (Interaction = Instruction) 
(Right) Selective model (Interaction = Selection)

Figure 11a Alienation of the network by intruders : 100 cycles with intrusion. Loss of the response to the front direction with an intrusion rate of 0.4 in the buffer of one of the two diff buffers.

Figure 11b Alienation of the eigenfrequency of the drive that responded to the right direction (0.25) in the experiment of figure 11a. 
FIGURE 1

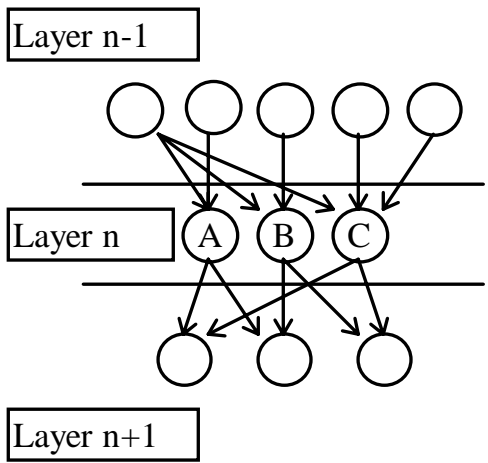

Figure 1 A 3-layer cellular structure 
FIGURE 2

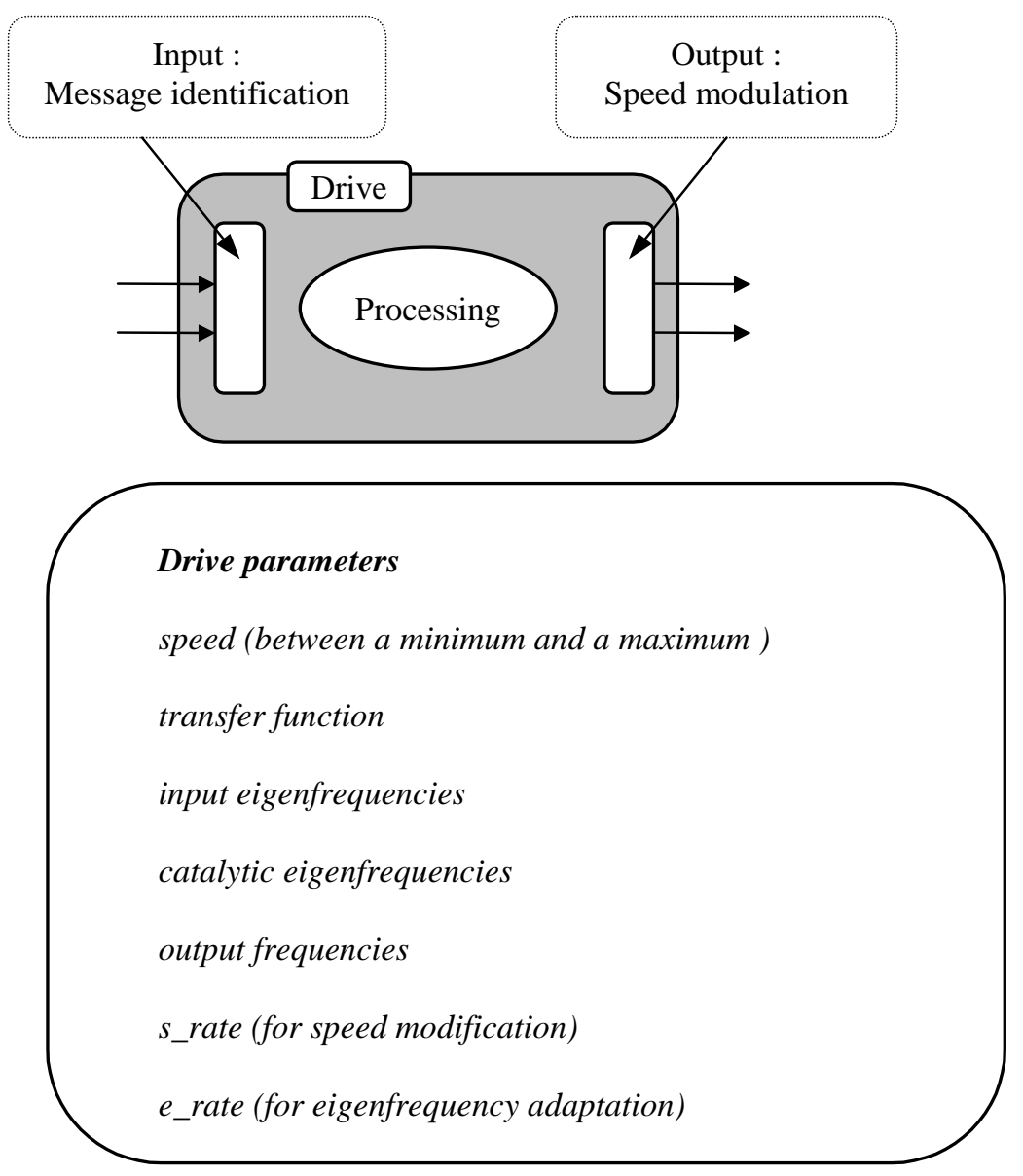

Figure 2 The drive model 


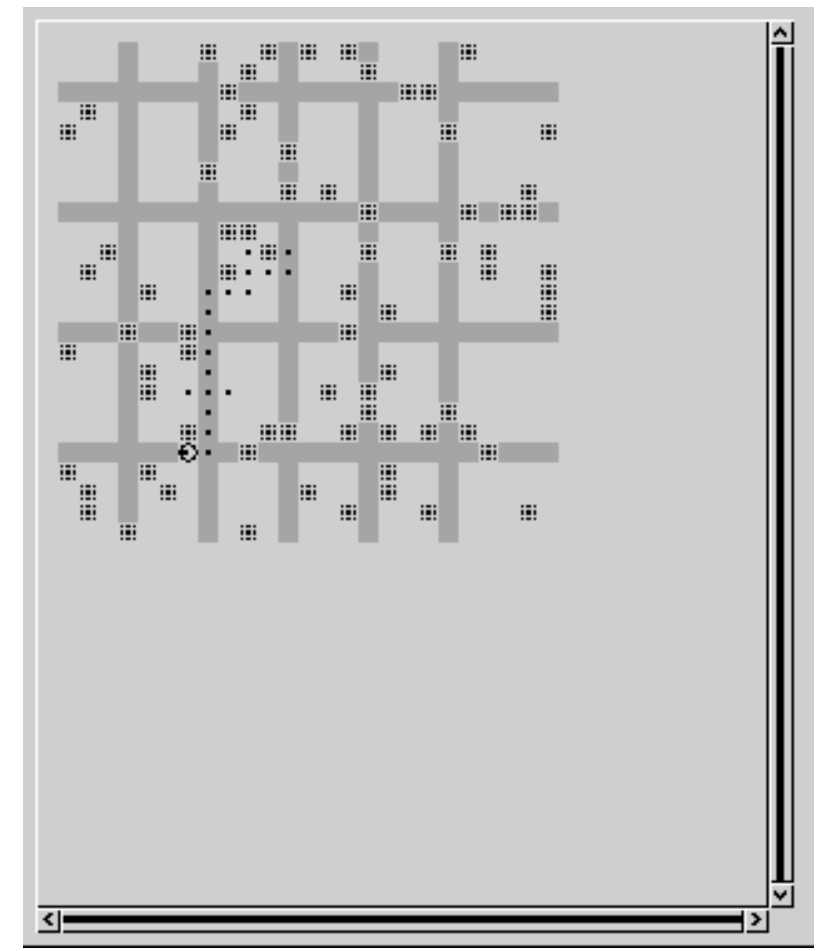

Figure 3 The agent in its navigation universe. Line following with simultaneous obstacle avoidance. The dark parts of the "surface" stand for the traces and the small grids stand for the obstacles. The dot line is the agent's trajectory. The deviations of the agent from the "optimal" trajectory are due to the presence of a degree of noise (here 10\%) in the generation of the line direction, what has as a consequence that the chosen direction is not always locally optimal ; this noise allows to the agent to avoid getting trapped in dead-ends. 
FIGURE 4

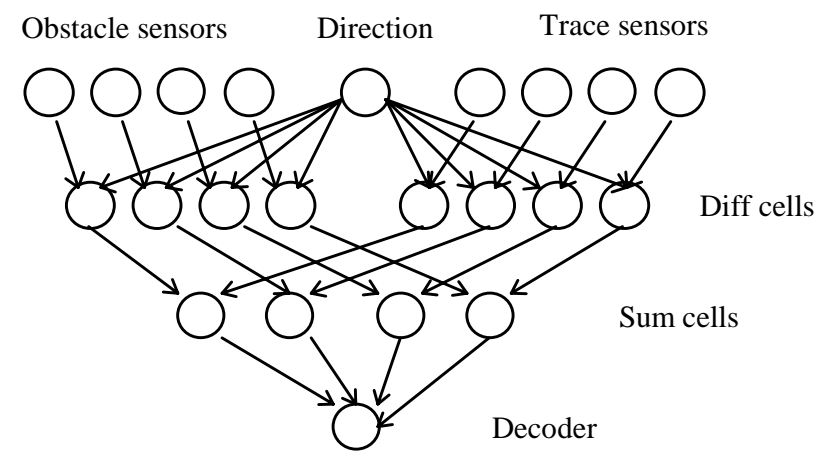

Figure 4 The algorithmic navigation system 
FIGURE 5

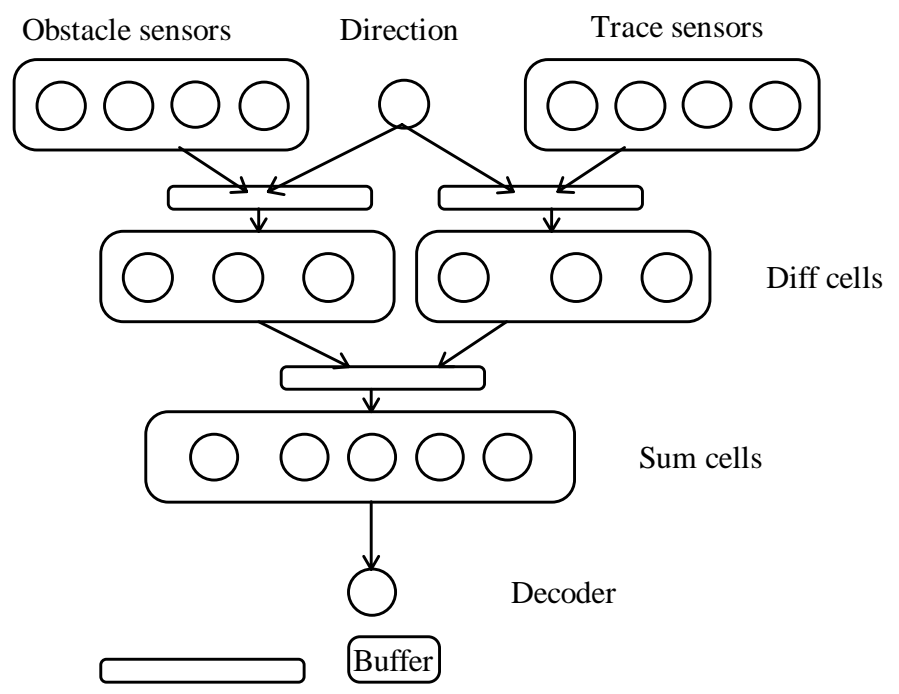

Figure 5 The self-organizing navigation system 
FIGURE 6

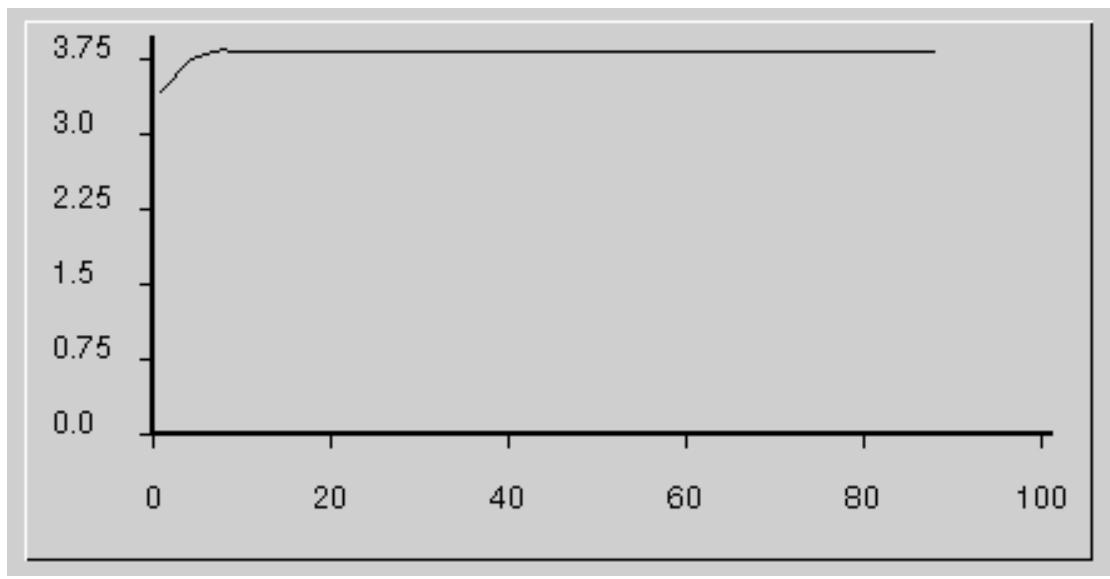

Figure 6 Total response speed stabilization. This speed is actually the total reaction speed of the agent, i.e., a measure internal to the agent, and does not necessarily represent a true speed as perceived by an external observer (cf. section 2.1). 
FIGURE 7

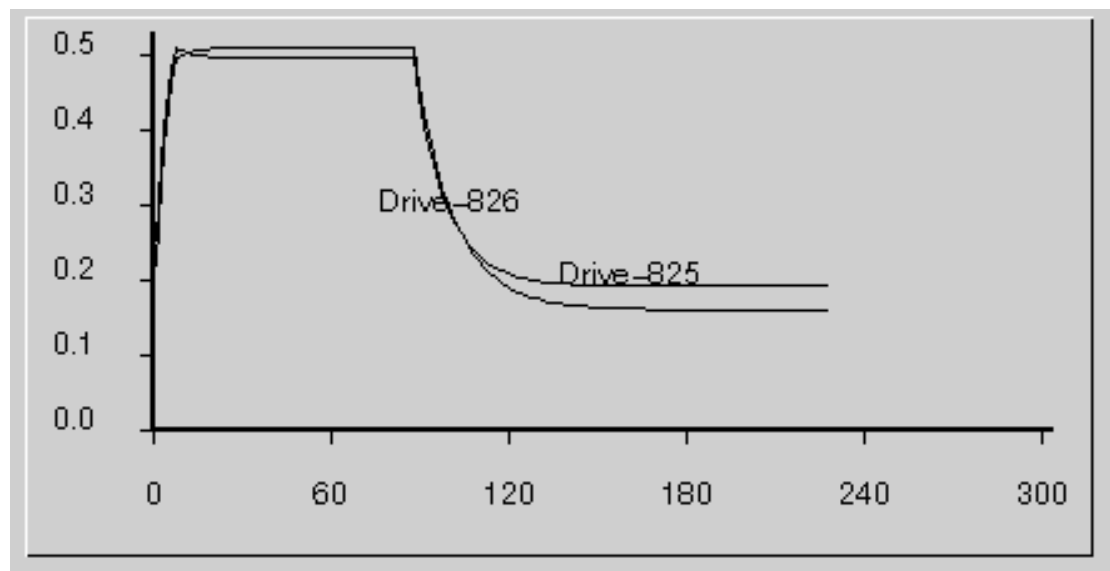

Figure 7 Drive speeds competition for a "diff" cell. In the first part of the curve the drives are stimulated, so their speeds rise toward their maximum ; in the second part, the drives are no longer stimulated and their speeds fall to their minimum. Note that the highest speeds of the two drives are not their maximal ones, they are away from 1.++ 


\section{FIGURE 8a}

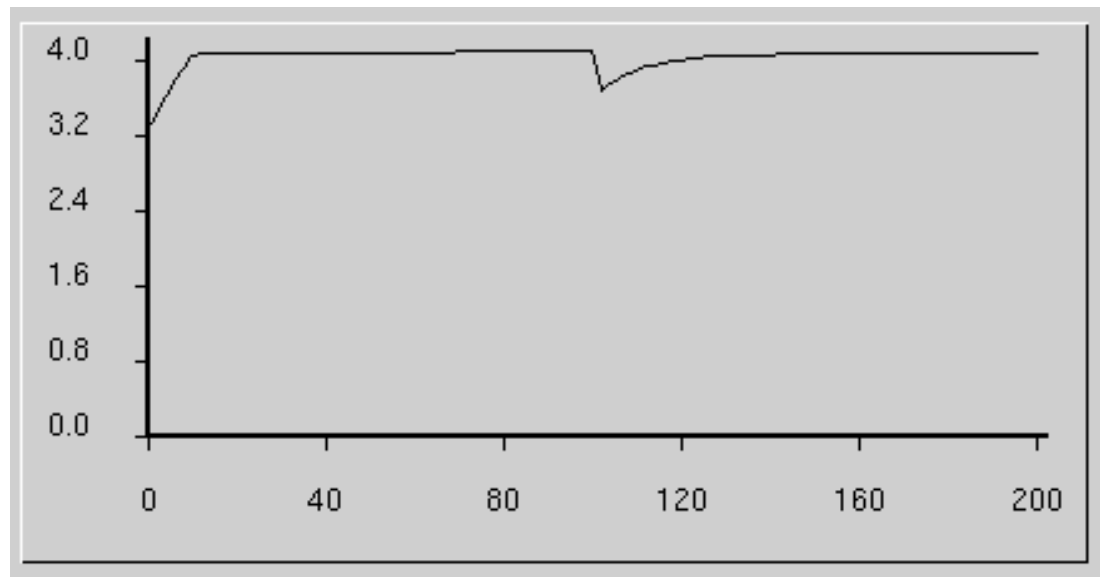

Figure 8a Re-stabilization of total speed. At $t=100$ removal of a diff cell that used to process the right direction $(0.25)$. The total speed decreases after the lesion and re-stabilizes later $(\mathrm{t}=200)$.

FIGURE $8 \mathrm{~b}$

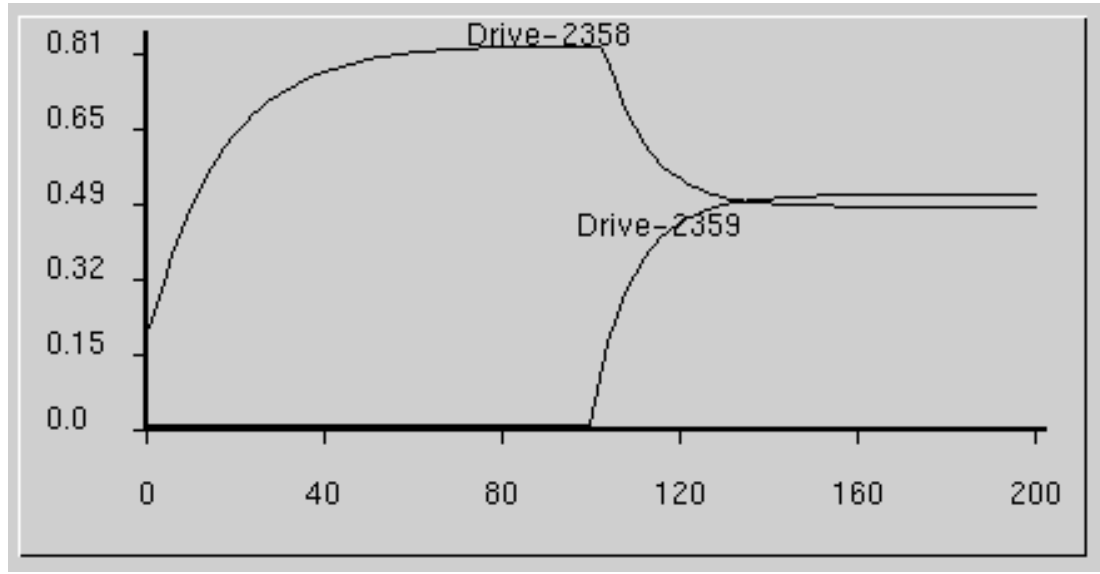

Figure 8b For the experiment of figure 8a, speed re-stabilization of two drives after the lesion $(\mathrm{t}=100)$ and the recovery. 
FIGURE 9

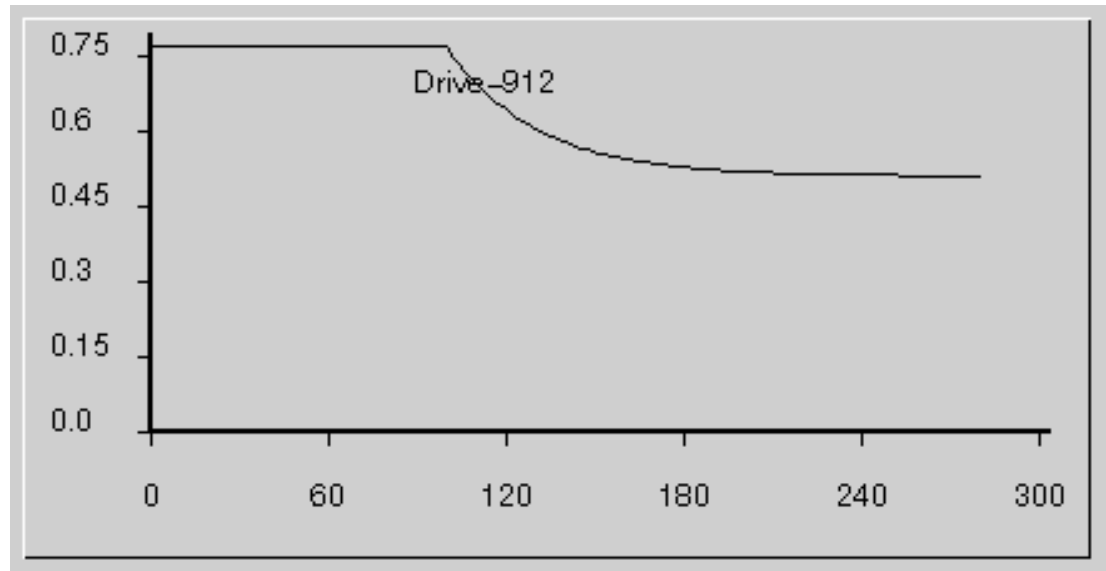

Figure 9 The response direction (or response identifier) of the drive that undertook the role of "front". 
FIGURE 10
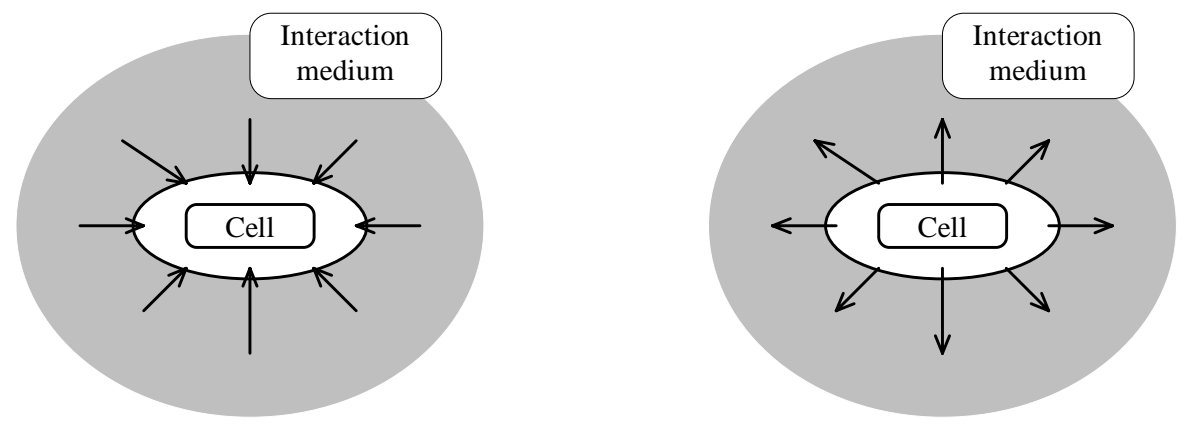

Figure 10

(Left) Instructive model (Interaction = Instruction)

(Right) Selective model (Interaction $=$ Selection) 


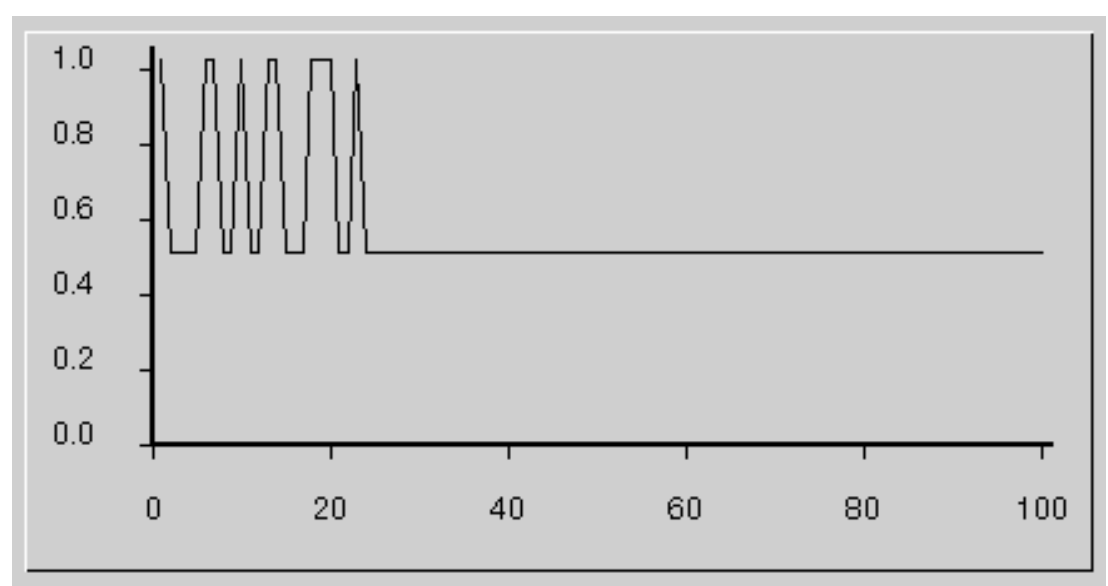

Figure 11a

Alienation of the network by intruders: 100 cycles with intrusion. Loss of the response to the front direction with an intrusion rate of 0.4 in the buffer of one of the two diff buffers.

FIGURE 18b

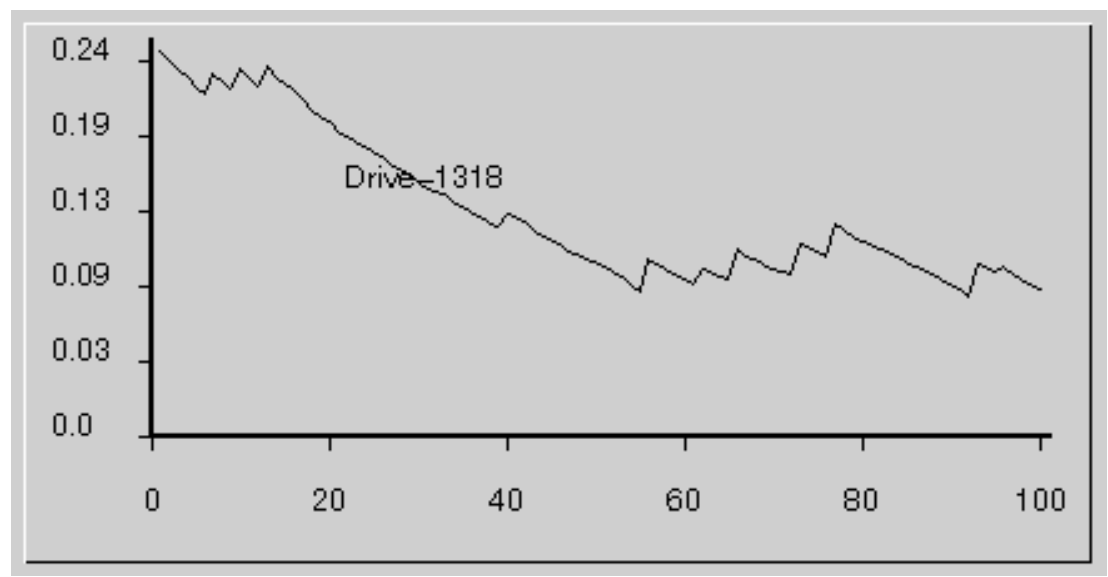

Figure 11b

Alienation of the eigenfrequency of the drive that responded to the right direction (0.25) in the experiment of figure $11 \mathrm{a}$. 


\section{TABLE LEGENDS}

Table 1 Recapitulation of the approach and the results 
TABLE 1

\begin{tabular}{|c|c|}
\hline The problem & Cellular organization of dynamic and fault-tolerant topology \\
\hline The application & A navigation problem \\
\hline The solution & $\begin{array}{l}\text { A "motivational" system (a system of drives executing in } \\
\text { parallel and in permanent competition with one another } \\
\text { for the consumption of the input messages) } \\
\text { Shared buffers instead of connections } \\
\text { Composite messages : pairs of values (identifier, data) } \\
\text { Consumable or catalytic messages } \\
\text { A competition mechanism (with the aid of reaction speeds) } \\
\text { Self-regulation of the dynamics of interaction with the world } \\
\text { (reaction speeds) } \\
\text { Developmental social adaptation : } \\
\text { Tit-for-tat "sociality" as far as recognition is concerned } \\
\text { Immune system of defense parallel to the "production" system } \\
\text { (recognition and elimination of foreign messages) }\end{array}$ \\
\hline The conclusions & $\begin{array}{l}\text { - Continuous self-organization } \\
\text { - Need for continuous developmental adaptation } \\
\text { - Network alienation by intruders and viruses (in the } \\
\text { absence of an immune system) } \\
\text { - Direct recognition of foreign messages } \\
\text { - Coupling between production system and immune system : } \\
\text { complementarity of the messages that are recognized by } \\
\text { - the two systems } \\
\text { - } \text { meed for direct aggressiveness in the network (the case of } \\
\text { - The role of diversity }\end{array}$ \\
\hline
\end{tabular}

